\title{
L'ACTIVATION COGNITIVE PRÉSOMMEIL ET L'EFFICACITÉ DU SOMMEIL CHEZ LES TRAVAILLEURS DE NUIT
}

Lorrie BEAUDOIN ${ }^{1, *}$, Alexandra REMON ${ }^{1}$, Camille MARTIN ${ }^{1}$, Michel BERTRAND ${ }^{1,2}$, Fatima BOUCHNAFA ${ }^{1,2}$, Séverine DE BILLY GARNIER ${ }^{1,2,3}$ \& Annie VALLIÈRES ${ }^{1,2,3}$

1 École de psychologie, Université Laval, Québec, Canada

${ }^{2}$ Centre de recherche CERVO, Québec, Canada

${ }^{3}$ Centre de recherche du CHU de Québec - Université Laval, Québec, Canada

*lorrie.beaudoin.1@ulaval.ca

\section{Pour citer l'article}

Beaudoin, L., Remon, A., Martin, C., Bertrand, M., Bouchnafa, F., De Billy Garnier, S., \& Vallières, A. (2018). L'activation cognitive présommeil et l'efficacité du sommeil chez les travailleurs de nuit. Psycause : Revue scientifique étudiante de l'École de psychologie de I'Université Laval, 8(2), 13-15. 


\section{L'ACTIVATION COGNITIVE PRÉSOMMEIL ET L'EFFICACITÉ DU SOMMEIL CHEZ LES TRAVAILLEURS DE NUIT}

Lorrie BEAUDOIN ${ }^{1}$, Alexandra REMON ${ }^{1}$, Camille MARTIN ${ }^{1}$, Michel BERTRAND ${ }^{1,2}$, Fatima BOUCHNAFA ${ }^{1,2}$, Séverine DE BILLY GARNIER ${ }^{1,2,3}$ \& Annie VALLIĖRES ${ }^{1,2,3}$

1 École de psychologie, Université Laval, Québec, Canada ${ }^{2}$ Centre de recherche CERVO, Québec, Canada ${ }^{3}$ Centre de recherche du CHU de Québec - Université Laval, Québec, Canada

\section{Introduction}

Au Canada, environ 30\% des travailleurs ont un horaire de travail non conventionnel, dont les travailleurs de nuit (Shields, 2002). Ces travailleurs suivent un processus veille-sommeil inversé, c.-à-d. qu'ils dorment le jour et restent éveillés la nuit. Dans ce contexte, les perturbations du sommeil sont caractérisées par une désynchronisation entre l'horaire de sommeil et le rythme circadien. Notons également l'effet de l'environnement de jour sur l'hygiène du sommeil considérant la quantité de bruit élevée, la présence de lumière et l'activation physiologique diurne. Certains paramètres caractérisant le sommeil en sont affectés négativement, incluant la latence d'endormissement (SOL), le temps total de sommeil (TST) et l'efficacité de sommeil (ES). Ces paramètres peuvent être calculés pour la période de sommeil principale ou considérant l'ensemble des périodes de sommeil sur 24 heures. Lorsqu'un tel phénomène est présent, il est question du trouble de sommeil lié à I'horaire de travail (THT). Ce trouble est principalement caractérisé par la présence de somnolence excessive ou de l'insomnie (American Academy of Sleep Medicine, 2014).

Jusqu'à maintenant, seul le dérèglement du rythme circadien est avancé pour expliquer le THT dans les études s'intéressant au travail de nuit. L'activation cognitive présommeil est pourtant rapportée dans la littérature comme étant étroitement liée à l'insomnie chez les travailleurs de jour (Harvey, 2002). L'activation cognitive se définit notamment par des pensées intrusives, qui sont des pensées ou des images mentales répétitives et indésirables. Les études portant sur les travailleurs de jour soutiennent que l'activation cognitive et les pensées intrusives ont pour effet de moduler I'ES et le TST à la baisse ainsi que le SOL à la hausse (McGowan, Behar \& Luhmann, 2016). Toutefois, les auteurs ne sont pas en mesure de statuer si un tel effet peut être généralisé au sommeil des travailleurs de nuit.

Le premier objectif de cette étude est de vérifier s'il existe une relation entre l'activation cognitive présommeil, les pensées intrusives et l'efficacité du sommeil chez les travailleurs de nuit présentant un THT. Le second objectif est de comparer l'influence de l'activation cognitive présommeil et des pensées intrusives chez les travailleurs de nuit souffrant d'un THT et chez les bons dormeurs (BD).

\section{Méthode}

Un total de 77 travailleurs de nuit en milieu hospitalier a été recruté avec une moyenne d'âge de 37,1 ans ( $E$ - $T=12,48)$. L'échantillon est composé de 60 femmes et 17 hommes. Les participants devaient être âgés d'au moins 18 ans et travailler un minimum de huit nuits par mois depuis au moins trois mois. Afin de statuer sur la présence de difficultés de sommeil, les participants ont été classifiés en deux groupes (THT et $\mathrm{BD}$ ) à partir des réponses à l'Entrevue diagnostique de l'insomnie adaptée aux travailleurs de nuit (Morin, 1993). Les participants éligibles ont rempli plusieurs questionnaires, dont I'Inventaire des pensées intrusives de Glasgow (Harvey \& Espie, 2004) et l'Échelle d'activation présommeil cognitive 
(Nicassio, Mendlowitz, Fussell \& Petras, 1985). Ceux-ci mesurent respectivement les pensées intrusives et l'activation cognitive présommeil. Un agenda du sommeil a été rempli par les participants sur une période de deux semaines pour mesurer les paramètres à chaque période de sommeil.

\section{Résultats et discussion}

La présente étude permet d'élargir les connaissances quant à l'activation cognitive présommeil et les pensées intrusives chez les travailleurs de nuit. Les résultats montrent que les pensées intrusives ne sont pas associées à un allongement de la latence d'endormissement principale ou sur 24 heures (voir Tableau 1). L'explication suggérée est que l'horaire de travail des travailleurs de nuit implique un dérèglement du cycle circadien et une forte pression homéostasique. Cette pression est fonction de la privation de sommeil et du temps écoulé depuis la dernière période de sommeil (Boivin \& Boudreau, 2014). Celle-ci permettrait de contrer les difficultés à initier le sommeil au coucher, et ce, peu importe le niveau d'activation cognitive. Les résultats indiquent également qu'une fréquence élevée de pensées intrusives est associée à un temps total de sommeil plus court pour la période principale de sommeil, ainsi qu'à une moins bonne efficacité du sommeil sur une période de 24 heures. Il est avancé que la quantité insuffisante de sommeil impliquerait surtout une difficulté à maintenir le sommeil en raison d'éveils précoces accentués par l'obligation de dormir de jour et les conditions environnantes défavorables. Au réveil, la pression homéostasique diminuée ne suffirait plus pour contrer les pensées intrusives. Parallèlement, les résultats impliquant l'activation cognitive présommeil se sont tous avérés non significativement corrélés aux paramètres de sommeil. II est envisagé qu'une forme plus générale d'activation n'est

\section{Tableau 1}

Matrice de corrélations entre l'activation cognitive (I'EAP-C), les pensées intrusives (I'IPIG) et les paramètres de sommeil chez les travailleurs de nuit présentant un THT.

\begin{tabular}{|c|c|c|c|c|c|c|c|c|}
\hline Mesures & 1 & 2 & 3 & 4 & 5 & 6 & 7 & 8 \\
\hline \multicolumn{9}{|l|}{ 1. Score à I'IPIG } \\
\hline 2. Score à I'EAP-C & $* 0,741^{a}$ & & & & & & & \\
\hline 3. SOL principal & $0,068^{b}$ & $0,225^{a}$ & & & & & & \\
\hline 4. SOL-24hres & $-0,163^{a}$ & $0,135^{b}$ & ${ }^{\star} 0,956^{\mathrm{a}}$ & & & & & \\
\hline 5. ES principale & $-0,161^{b}$ & $0,008^{b}$ & $*-0,447^{a}$ & $*_{-0} 0,471^{a}$ & & & & \\
\hline 6. ES-24hres & $*_{-0} 0,278^{b}$ & $-0,144^{b}$ & $*-0,345^{\mathrm{a}}$ & $*-0,452^{\mathrm{a}}$ & $\star 0,751^{a}$ & & & \\
\hline 7. TST principal & ${ }^{*}-0,338^{a}$ & $-0,073^{a}$ & $0,245^{\mathrm{a}}$ & $0,203^{a}$ & ${ }^{\star} 0,397^{a}$ & ${ }^{\star} 0,287^{a}$ & & \\
\hline 8. TST-24hres & $-0,259^{a}$ & $-0,099^{a}$ & $0,129^{a}$ & $0,036^{a}$ & $\star 0,466^{a}$ & ${ }^{\star} 0,433^{a}$ & ${ }^{\star} 0,729^{a}$ & \\
\hline
\end{tabular}

Note. IPIG = Inventaire des pensées intrusives de Glasgow. EAP-C = Échelle d'activation présommeil (sous-échelle cognitive). SOL principal = Latence d'endormissement pour la période principale de sommeil. SOL-24hres = Latence d'endormissement pour l'ensemble des périodes de sommeil sur 24 heures. ES principale = Efficacité du sommeil pour la période principale. ES-24hres = Efficacité du sommeil pour l'ensemble des périodes de sommeil. TST principal $=$ Temps total dormi pour la période principale. TST-24hres $=$ Temps total dormi pour l'ensemble des périodes de sommeil.

* $p<0,05$

a. Corrélation unilatérale de Pearson $(r)$

b. Corrélation unilatérale de Spearman $(\rho)$ 
pas suffisante pour entraîner des difficultés de sommeil auprès des travailleurs de nuit, en comparaison avec des formes spécifiques d'activation comme les pensées intrusives. Par ailleurs, les résultats montrent que les travailleurs du groupe THT font davantage l'expérience de pensées intrusives et d'activation cognitive présommeil comparativement au groupe de BD (voir Figure 1). La présence d'activation cognitive semble donc liée au diagnostic du THT

En définitive, les résultats provenant d'études s'intéressant aux travailleurs de jour ne peuvent être généralisés de facto aux travailleurs de nuit considérant entre autres les corrélations non significatives quant à la latence d'endormissement. De futurs travaux auraient ainsi avantage à préciser le rôle des diverses formes d'activation cognitive dans le THT.

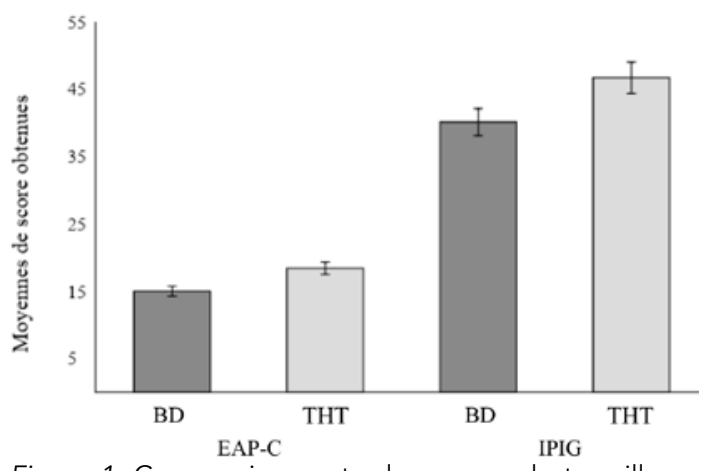

Figure 1. Comparaison entre le groupe de travailleurs de nuit bons dormeurs et THT pour l'activation cognitive présommeil (I'EAP-C) et les pensées intrusives (I'IPIG). Les barres d'erreurs correspondent au seuil de signification alpha de $5 \%$.

\section{Références}

American Academy of Sleep Medicine. (2014). International classification of sleep disorders. Darien, IL : American Academy of Sleep Medicine.

Boivin, D. B., \& Boudreau, P. (2014). Impacts of shift work on sleep and circadian rhythms. Pathologie-Biologie, 62, 292 301. doi:10.1016/j.patbio.2014.08.001

Harvey, A. G. (2002).Acognitifmodel ofinsomnia. Behaviour Research and Therapy, 40, 869893. doi:10.1016/S0005-7967(01)00061-4

Harvey, K. J., \& Espie, C. A. (2004). Development and preliminary validation of the Glasgow Content of Thoughts Inventory (GCTI): A new measure for the assessment of presleep cognitive activity. The British Journal of Clinical Psychology, 43, 409-420. doi:10.1348/0144665042388900

McGowan, S. K., Behar, E., \& Luhmann, M. (2016). Examining the relationship between worry and sleep: A daily process approach. Behavior Therapy, 47, 460-473. doi:10.1016/j.beth.2015.12.003

Morin, C. M. (1993). Insomnia: Psychological assessment and management. New York, NY: Guilford Press.

Nicassio, P. M., Mendlowitz, D. R., Fussell, J. J., \& Petras, L. (1985). The phenomenology of the pre-sleep state: The development of the pre-sleep arousal scale. Behaviour Research and Therapy, 23, 263-271. doi:10.1016/0005-7967(85)90004-X

Shields, M. (2002). Shift work and health. Health Reports, 13(4), 11-33. 